\title{
Papillary thyroid microcarcinoma presenting as lymph node metastasis - a diagnostic challenge: case report and systematic review of literature
}

\author{
Athanasios D. Anastasilakis, ${ }^{1}$ Stergios A. Polyzos, ${ }^{2}$ Polyzois Makras, ${ }^{3}$ Lampros Kampas, ${ }^{4}$ \\ Rosalia-Maria Valeri, ${ }^{5}$ Kyriakoulis Dimitrios, ${ }^{6}$ Thomas Zarampoukas, ${ }^{7}$ Maria Alevizaki ${ }^{8}$ \\ ${ }^{1}$ Department of Endocrinology, 424 General Military Hospital, ${ }^{2}$ Second Medical Clinic, Aristotle University of \\ Thessaloniki, Ippokration General Hospital, Thessaloniki, ${ }^{3}$ Department of Endocrinology and Diabetes, 251 Hellenic \\ Air Force \& VA General Hospital, Athens, ${ }^{4}$ Department of Cytopathology, 424 General Military Hospital, Thessaloniki, \\ ${ }^{5}$ Department of Cytopathology, Cancer Hospital "Theagenion", ${ }^{6}$ Department of Ultrasonography, 424 General Military \\ Hospital, ${ }^{7}$ Department of Pathology, Aristotle University of Thessaloniki, Thessaloniki, ${ }^{8}$ Endocrinology, Metabolism \\ and Diabetes Unit, Department of Medical Therapeutics, Alexandra Hospital, Athens, Greece
}

\begin{abstract}
Papillary thyroid microcarcinomas (PTMCs) have an excellent prognosis, although a few may metastasize to cervical lymph nodes. However, an infiltrated palpable neck node without evidence of thyroid disease at presentation is uncommon. We report a patient with PTMC presenting as a solitary lymph node metastasis without evidence of primary thyroid tumor in thyroid imaging and with inconclusive lymph node fine-needle biopsy (FNB) cytology. In our case, node excision and histological examination set the diagnosis and immunocytochemical staining of the FNB specimens verified it. A systematic review of reported similar cases was performed; relevant diagnostic dilemmas were also summarized. The clinical presentation of this type of papillary carcinoma becomes evident at a relatively younger age and affects almost equally the two genders; the enlarged lymph node is almost exclusively ipsilateral to the primary tumor, which may be unifocal or multifocal and is difficult to detect by thyroid imaging modalities. Lymph node FNB cytology, thyroglobulin (Tg) measurement in the washout liquid of the FNB needle, FNB immunocytochemistry and lymph node excision accompanied by histological examination provide a stepwise diagnostic approach. We conclude that PTMC may present as a lymph node metastasis without evidence of a primary thyroid tumor. In such cases, thyroid malignancy should be suspected and, in the presence of negative or non-diagnostic lymph node FNB cytology, measurement of $\mathrm{Tg}$ in the fluid aspirate should be performed.
\end{abstract}

Key words: Fine-needle biopsy, Immunocytochemistry, Lymph node, Metastasis, Papillary, Thyroid cancer 


\section{INTRODUCTION}

Papillary thyroid carcinoma (PTC) may metastasize to cervical lymph nodes. However, the presence of an infiltrated palpable neck node without evidence of apparent thyroid nodular disease at presentation is uncommon. ${ }^{1}$

Thyroid carcinomas of a maximum diameter of $10 \mathrm{~mm}$ or less are defined as microcarcinomas; they are usually found incidentally (thyroid incidentalomas) and the majority of them are papillary thyroid microcarcinomas (PTMCs). Most PTMCs have an excellent prognosis, but a few present with palpable lymphadenopathy; ${ }^{2}$ even in very small PTMCs with a maximum diameter less than $1 \mathrm{~mm}$, nodal metastases have been described. ${ }^{3}$ Although a multifactorial regression model is lacking in the literature, an apparent lymph node metastasis (LNM) may be associated with increased aggressiveness of the tumor. Indeed, patients with LNMs are at risk for nodal recurrence, but this is not associated with higher mortality. ${ }^{4}$

Cervical LNMs as the single manifestation of PTC are extremely rare. ${ }^{5}$ The LNMs may be solitary or multiple and they often appear cystic in ultrasonography ${ }^{1,5-11}$ because of subcortical liquefaction resulting in a discrete fluid-filled neck mass. ${ }^{12}$ The occult primary thyroid malignancy can also be solitary or multifocal. ${ }^{8,5}$ LNMs may be ipsilateral or bilateral and appear to be more frequent in patients with multifocal primary tumors. ${ }^{13-15}$

Fine-needle aspiration biopsy (FNB) cytology represents a useful tool in the differential diagnosis of a neck mass of unknown origin. It has been ascertained that thyroglobulin $(\mathrm{Tg})$ measurement in the washout liquid of the needle used for FNB (FNB-Tg) of a neck mass can help distinguish neck masses of thyroid origin from other masses. ${ }^{16}$

Herein, we report a patient with multifocal PTMC, presenting as a solitary ipsilateral lymph node metastasis, with no evidence of thyroid disease on thorough thyroid imaging. A systematic review was subsequently performed; previously reported similar cases as well as relevant diagnostic dilemmas are summarized.

\section{PATIENT REPORT}

A 41-year-old woman was referred to our hospital for evaluation of a right lateral cervical mass, which had been noticed 4 months before presentation and had not subsided with broad spectrum antibiotics. The patient was clinically euthyroid, with an apparently solitary palpable mass of approximately $25 \mathrm{~mm}$ on the right supraclavicular fossa. The mass was firm, mobile and painless on physical examination. She reported no personal or family history of thyroid disease, no sweating or diarrhea and no concomitant medications. The thyroid function tests and serum calcitonin were within normal range, while thyroidperoxidase autoantibodies (anti-TPO) were negative and anti-thyroglobulin autoantibodies (anti-Tg) were strongly positive $(520 \mathrm{IU} / \mathrm{ml}$, normal $<4.1)$. Serum $\mathrm{Tg}$ was not measured because it was considered that cross reactivity with anti-Tg could result in an inaccurate value; furthermore, it is not recommended in the diagnosis of thyroid carcinoma. ${ }^{17}$

Ultrasound (US) examination confirmed the presence of a 27 x $13 \mathrm{~mm}$ solid mass, which was strongly hypoechoic, heterogeneous, with no presence of hilum and with several microcalcifications and an area of cystic degeneration. Doppler ultrasound indicated increased central blood flow. The lesion resembled an abnormal lymph node. Several enlarged lymph nodes with benign ultrasound characteristics were also observed. The thyroid gland was normal in size with mild hypogenicity and heterogeneity but without any apparent nodules within the thyroid parenchyma.

To determine the origin of the lesion, an ultrasound-guided FNB was performed in the neck mass using a $23 \mathrm{G}$ needle. The specimens were received fresh and were prepared using the ThinPrep technique (Liquid Based - Cytyc Corp., Boxborough, Massachusetts, U.S.A.) and stained by the Papanicolaou method. Cytological analysis showed lymphoid elements mixed with polymorphous epithelial cells with atypical nuclei, suggesting lymph node metastasis from a malignancy of epithelial origin. Subsequent immunocytochemistry (ICC) examination of the specimens was positive for Cytokeratin-7 (CK7) and negative for Cytokeratin-20 (CK20), indicating as possible primary sites the breast, lung, ovaries (endometrial and non-mucinous carcinomas), thyroid and stomach, and excluding other primary sites, such as the urinary tract and colon. The examination was negative for calcitonin and weakly positive for Tg. 
After excision and histological examination of the lymph node, the diagnosis of metastatic PTC was established. We retrospectively performed further ICC markers [Thyroid Transcription Factor-1 (TTF1), Cytokeratin-19 (CK19), CD44 and HBME-1] in preserved, unused cytological specimens; all these markers were expressed in the specimens, confirming the diagnosis.

Based on the cytology/histology results, we conducted a Computed Tomography (CT) scan followed by Magneting Resonance Imaging (MRI) of the neck, which revealed several enlarged supraclavicular and jugular chain lymph nodes bilaterally, but no evidence of the primary tumor.

Subsequently, the patient was subjected to total thyroidectomy and bilateral comprehensive neck dissection. Multiple foci ( 3 in the right lobe, 1 in the isthmus and 1 in the left lobe) of follicular variant PTMC with a diameter of $2-5 \mathrm{~mm}$ were found on thyroid histological examination. There was no evidence of extrathyroidal invasion. Surprisingly, except for the originally excised right cervical node, all the remaining excised lymph nodes $(n=39)$ were disease-free. The patient is currently awaiting ablative therapy with iodine.

\section{LITERATURE SEARCH METHOD}

A computerized advanced search, not limited by publication time and not restricted to the Englishlanguage literature, was performed in PubMed. Afterwards, the search was extended to the "Related Articles" of each selected article in PubMed and its references. Finally, automatic alerts were activated in PubMed to add relevant articles published after the initial search. In the cases of unavailability of the paper or language barrier, we attempted to contact the authors and retrieve missing information.

Patients reported in the included studies should fulfill the above criteria: 1) no history of thyroid nodular disease; 2) initial presentation as a random cervical lymph node enlargement of unknown origin; 3 ) histological diagnosis of papillary thyroid carcinoma.

\section{LITERATURE SEARCH RESULTS}

The original query provided 155 potentially rel- evant journal articles. After excluding reviews, editorials, hypotheses, experimental and in-vivo studies, 59 potentially relevant articles remained, which were evaluated in detail. Twelve of them were finally selected, whereas the remaining 47 were excluded, for the following reasons: the thyroid nodule(s) was/were diagnosed before the lymph nodal metastasis $(n=4)$; the initially observed metastasis was other than cervical lymph nodal $(\mathrm{n}=10)$; the primary thyroid carcinoma was medullary or sclerosing $(\mathrm{n}=7)$; the primary focus was extra-thyroid $(n=4)$; the article was related to other disease or coexistence of thyroid carcinoma with other disease $(n=14)$; the article was related to diagnostic or surgical management of thyroid carcinoma $(n=6)$; we could not retrieve the full-text article or communicate with authors $(n=2){ }^{20,21}$

Furthermore, 16 articles were retrieved after searching the "Related Articles" link next to each selected article in PubMed and the references of each selected article. Finally, automatic alert up to 15 March 2012 provided no more articles eligible for the systematic review. The final number of eligible reports in this systematic review besides our case was 28 .

All of the retrieved articles were of level 3 of evidence, leading mainly to grade $\mathrm{C}$ of recommendations, since they were case reports, case series or observational studies. ${ }^{22}$

Patient characteristics and diagnostic procedures of all retrieved cases are summarized in Table 1. Studies not reporting detailed clinical or diagnostic management were excluded from the Table. ${ }^{2,15,23}$ For the purpose of completeness, all studies describing a metastatic lymph node of thyroid origin without evidence of a thyroid primary focus at initial presentation are displayed in Table 1, regardless of the size of the primary focus (earlier definition of occult thyroid carcinoma required a less than $15 \mathrm{~mm}$ maximum diameter; furthermore, some authors had reported as occult even greater carcinomas, when they were not clinically evident at presentation). At the end of Table 1, cumulative descriptive statistics for the sum of patients as well as for those fulfilling the strict current criteria of PTMC are presented.

\section{DISCUSSION}

Lateral cervical lesions are usually benign lesions, 


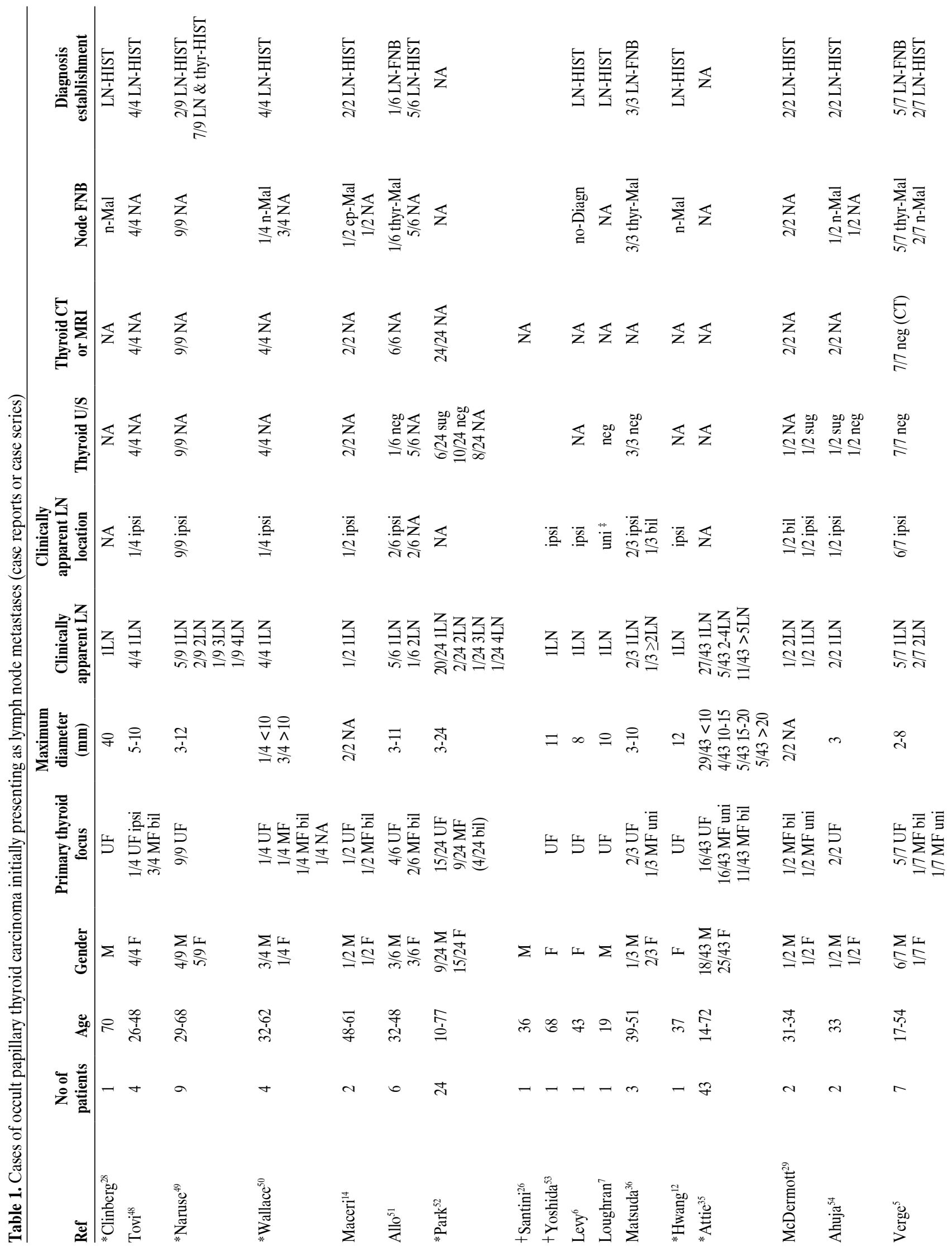




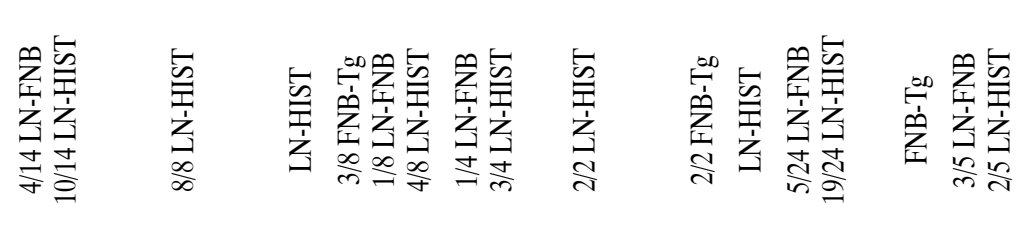

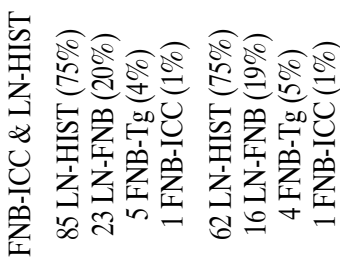

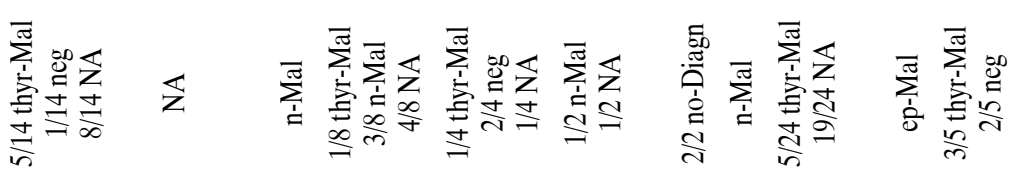

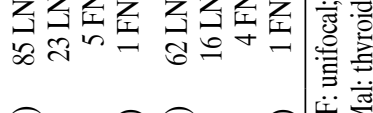

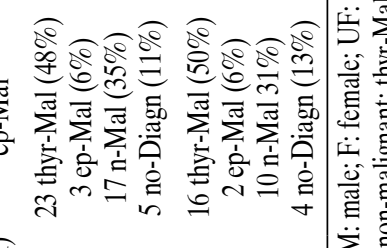

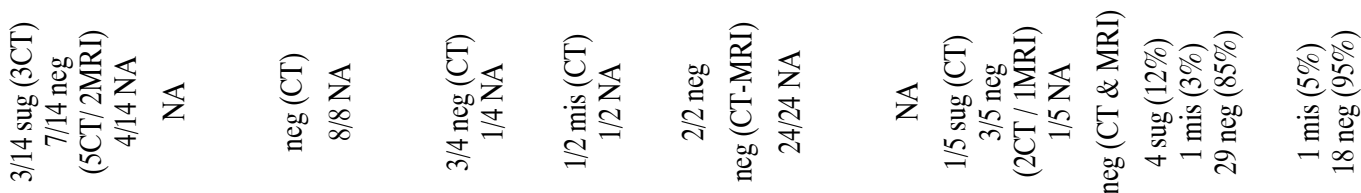

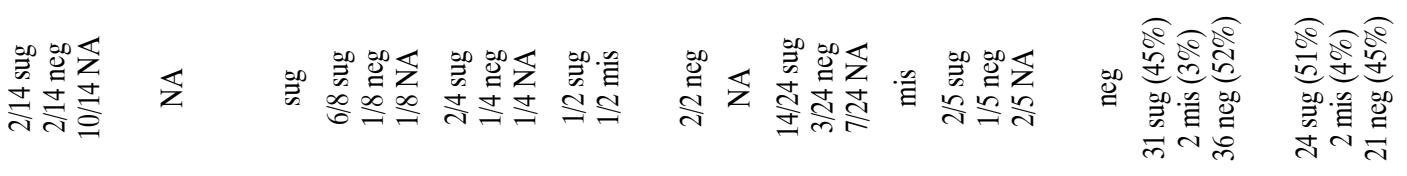

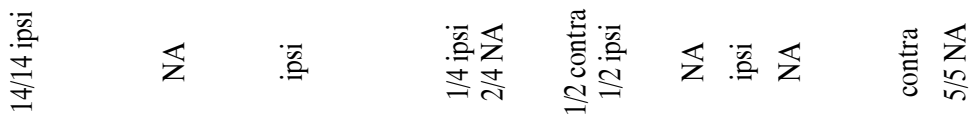

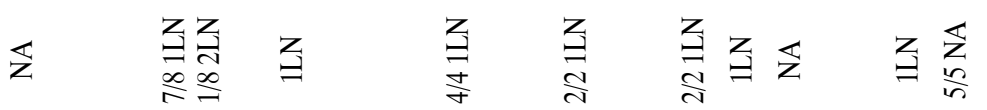

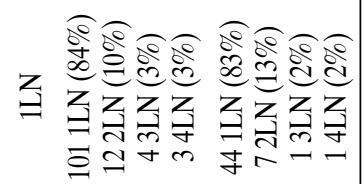

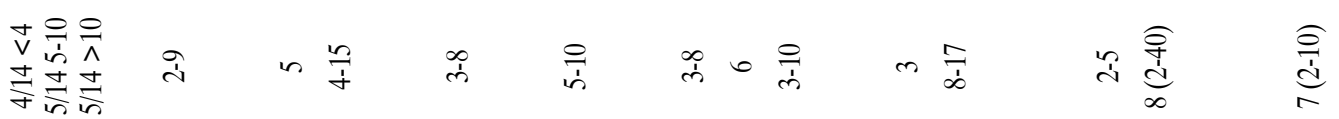

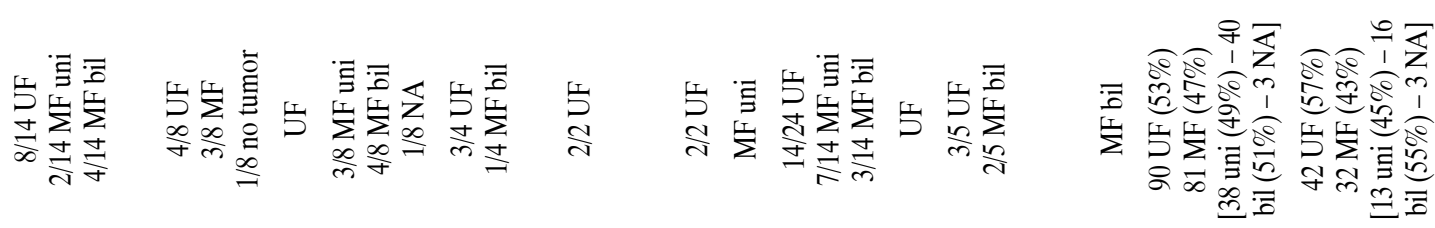

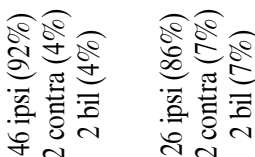

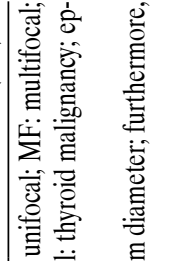

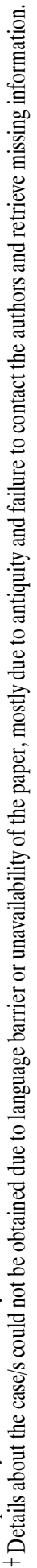

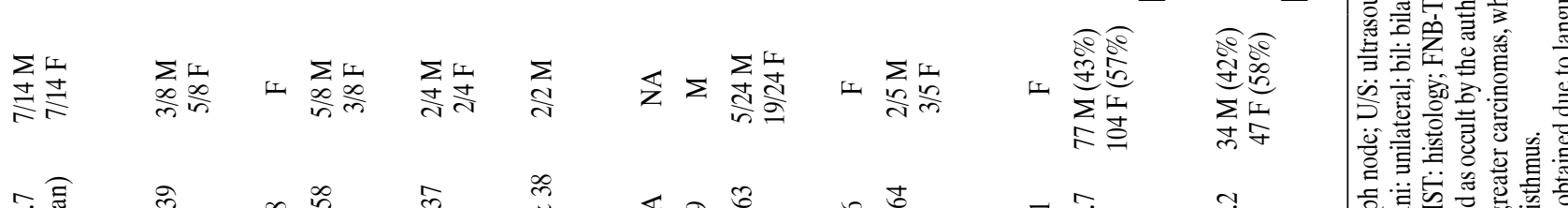

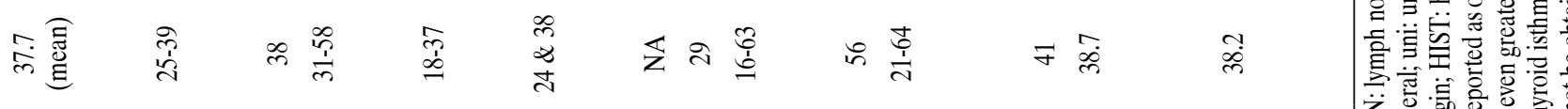

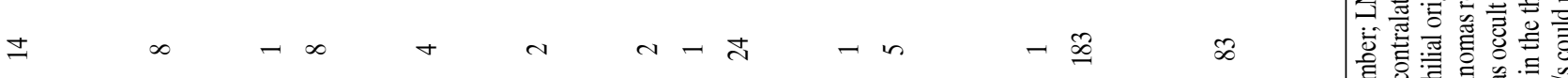

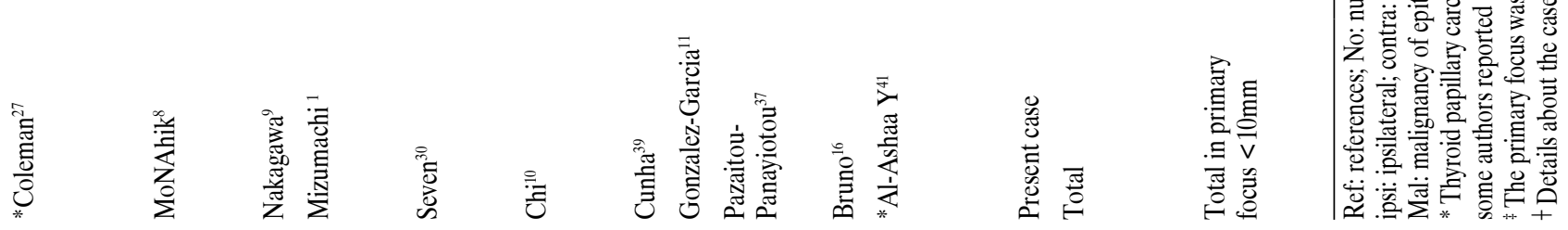


mainly branchial cysts, occurring predominantly in young people. Malignant lateral cervical lesions are less frequent and can be caused by tumors arising mainly from the oronasopharyngeal area, thyroid, lungs and salivary glands. ${ }^{5}$ In this setting, thyroid malignancy should be foremost considered, even in the absence of thyroid disease, as PTC has the highest prevalence. ${ }^{24}$ Indeed, the risk of a LNM from an unrecognized PTC or PTMC is considerable ${ }^{25,17}$ while follicular, medullary and sclerosing neoplasms may also become evident as a LNM. ${ }^{26,27}$ By contrast, it is unlikely for a primary salivary gland or a oronasopharyngeal neoplasm to manifest as a cervical LNM without clinical ${ }^{28}$ or imaging ${ }^{11}$ evidence of the primary site mass.

PTC typically presents at a relatively younger age compared to the rest of these malignancies. It has been proposed that in patients less than 40 years of age presenting with a cervical lateral mass, thyroid malignancy should be one of the first diagnoses to be considered. ${ }^{29,30}$ Indeed, mean age of the herein reviewed cases was less than 40 years (Table 1), while PTMC without a clinically evident LNM presents at a mean age of 45-52 years. ${ }^{2,15,23,31}$ These differences probably reflect the more aggressive behavior of PTMCs presenting as LNMs, which may arise at a similar age but become apparent earlier than the remaining PTMCs.

Thyroid cancer in general ${ }^{32}$ and PTC in particular ${ }^{5}$ are more common in women, with a reported ratio of 3:1; in PTMC series, females represent 70-89\% of the cases. ${ }^{2,15,23,31}$ However, in cases of PTMC presenting as an enlarged lymph node, female predominance is considerably lower (Table 1). This may also be related to the abovementioned aggressiveness of PTMCs presenting as LNM, as PTC is more aggressive in men. ${ }^{33}$

The majority of PTCs are unifocal. However, LNMs appear to be more frequent in patients with multifocal PTCs. ${ }^{13-15}$ We identified an almost equal incidence of unifocal and multifocal tumors in patients presenting with a clinically apparent LNM (Table 1). Among patients with multifocal thyroid tumor, bilateral disease was slightly more frequent than unilateral localization. This is in contrast with most studies in patients with PTMCs without clinically evident LNMs, which report a slight predominance of multifocal unilateral over multifocal bilateral le- sions. ${ }^{2,15,23,31}$ Once more, the aggressiveness of the PTMCs presenting as LNMs could be the reason for this discrepancy.

Thus, data from our review reveal several risk factors for a LNM in the case of a PTMC, including younger age, male gender and multifocal and bilateral primary thyroid lesions. Similar results were recently reported by Zhang et al. ${ }^{34}$

A LNM as the initial presentation of PTC is not so rare; it has been reported to be between $5.2 \%{ }^{35}$ and $13.6 \%{ }^{14}$ of all PTCs. In the majority of the patients presented in Table 1, the clinically evident node/s was/were ipsilateral to the primary tumor, with the exceptions being mostly cases with multifocal PTMC. However, the exact location of the primary tumor (upper, middle or lower part of the lobe) cannot predict the group of lymph nodes infiltrated by PTMC (supraclavicular, jugular, pretracheal, etc.). ${ }^{2}$

Besides the enlarged node initially observed, several other nodes are often found infiltrated after neck dissection. ${ }^{5,36,37}$ In our case, however, only the initially observed lymph node was infiltrated, despite the multifocal thyroid lesions. To our knowledge, this has not been previously reported, at least within the retrieved cases or case series.

Although in theory extrathyroidal invasion would be expected to be more frequent in patients presenting with an enlarged lymph node, there are no relevant data in many of the reports included in this review. Thus, we cannot draw safe conclusions regarding such an association. Moreover, there are no data in the literature concerning associations of lymph node size with aggressiveness. In the recent study of Zhang et al, extrathyroidal extension was associated with both central and lateral LNM. ${ }^{34}$

In all cases shown in Table 1 thyroid lesions were not palpable on physical examination, while imaging procedures, including thyroid ultrasound, neck CT and MRI, seem to have low (well below 50\%) sensitivity in detecting occult thyroid tumors (Table 1). This is in accordance with other studies reporting that the abovementioned diagnostic modalities were usually ineffective in locating the primary lesion. ${ }^{11,14,27}$ In these cases, histological examination of the excised thyroid revealed the primary focus/foci. However, there are very few cases in the literature, of PTC lying 
in a lateral cervical cyst with no occult primary tumor identified within the thyroid; in these cases, malignancy is attributed to neoplastic transformation of ectopic thyroid tissue lying within the branchial cyst. ${ }^{38}$

US examination in particular is generally considered a useful diagnostic tool for the study of both the thyroid gland and a cystic mass in the neck; ${ }^{17}$ tumors as small as $1 \mathrm{~mm}$ in diameter can be detected. However, thyroid US findings were normal or misleading in about half of the reviewed patients (Table 1). Likewise, US of the enlarged neck mass cannot clearly distinguish cystic LNMs from branchial cleft cysts. ${ }^{5}$ An irregularly thick wall, internal debris and the presence of solid echogenic components adherent to the wall point toward a possible diagnosis of malignancy. ${ }^{10}$

FNB cytology of a cervical lymph node has been proposed as a very useful procedure to discover occult thyroid carcinoma lying within the node. ${ }^{36}$ However, other studies have reported $29 \%$ to $43 \%{ }^{39}$ and even $50 \%$ of non-diagnostic or false negative results in FNB of the affected lymph node, as shown by the cumulative data presented in Table 1 . The possibility of false negative diagnosis is even higher in the case of cystic degeneration of the $\mathrm{LNM}^{39}$ because the classical pathologic features of PTC, such as papillae and psammoma bodies, may be sparse or not present, and nuclear abnormalities may be the only finding. ${ }^{8}$ Therefore, negative FNB cytology results cannot safely exclude the thyroid origin of the lesion.

In ours and a few other cases, ${ }^{14,16}$ FNB cytology revealed epithelial malignancy but could not define the origin of the tumor cells. In this setting, imaging with CT or MRI does not seem to help locate primary lesions in the thyroid and their role should be limited to disease staging. Positron emission tomography (PET)-CT might have a role in the localization of the primary tumor, although evidence is currently limited. ${ }^{40}$ FNB-Tg has been reported to be more sensitive than FNB cytology in differentiating LNMs from PTMCs. ${ }^{16,39}$ Macroscopically, the aspirates from such lesions are often, but not always, red or brown in color because of the presence of $\mathrm{Tg} .{ }^{41}$ However, this technique requires acquisition of a relatively liquid specimen, which is more feasible in nodes with cystic degeneration rather than in solid nodes, as in our case, where solid cytologic material with abundant cells was obtained; furthermore, the result may be affected by the presence of anti-Tg, which were positive in our patient. Finally, FNB-Tg may be false negative in an anaplastic thyroid tumor or an undifferentiated PTC. On the other hand, ICC may help distinguish PTM from other epithelial neoplasms in solid specimens without interference with anti-Tg and in undifferentiated forms of thyroid malignancy, although it must be noted that ICC has a considerably higher cost than FNB-Tg and, therefore, should be used in the event of inconclusive FNB cytology. Some authors believe that FNB cannot replace histological diagnosis and an excision biopsy should be performed for both diagnostic and therapeutic purposes ${ }^{10}$ however, ICC of the FNB specimens is much easier and less invasive. In lymph nodes smaller than $15 \mathrm{~mm}$, analysis for thyroid specific genes can be useful in identifying thyroid metastasis. ${ }^{42}$ The American Thyroid Association / European Thyroid Association / Associazione Medici Endocrinologi recent guidelines recommend the measurement of $\mathrm{Tg}$ (and calcitonin) in the washout of the needle used for FNB of a cervical neck mass. ${ }^{17}$

Especially ICC seems to play a key role in the accurate cytodiagnosis in cases of metastatic neoplasms with unknown primary site. The ThinPrep technique offers an easier application of ICC due to the creation of archival material; additionally, ICC consumes fewer reagents due to the lower background and smaller area of the ThinPrep slide containing the target cells. ${ }^{43} \mathrm{In}$ our case, besides $\mathrm{Tg}$, we used a panel of additional specific immunomarkers: TTF-1, a tissue-specific factor expressed in normal lung and thyroid tissue, ${ }^{44}$ CK19, an immunomarker used to discriminate papillary from other thyroid malignancies ${ }^{45} \mathrm{CD} 44$, which is expressed in $88 \%$ of classic PTC, ${ }^{46}$ while HBME1 distinguishes benign from malignant thyroid lesions. ${ }^{47}$ None of the above markers provides a $100 \%$ accurate diagnosis of PTC; however, the combination of positive Tg, TTF-1 and CK19 seems to provide the diagnosis of metastatic PTC with satisfactory accuracy.

In conclusion, a patient with PTMC presenting as LNM with no evidence of primary thyroid tumor in thyroid imaging and inconclusive lymph node FNB cytology is reported. In such cases, thyroid malignancy should be excluded. The proposed stepwise diagnostic approach includes: i) Tg measurement in the washout liquid of the FNB needle, ii) ICC examination of the FNB smears for Tg, TTF-1 and CK19 in the presence 
of positive anti-Tg or in rather solid material or suspicion of undifferentiated thyroid malignancy and iii) lymph node excision and histological examination if the above two methods fail to provide definite diagnosis. When the diagnosis is established, imaging of the neck and the chest should be performed to identify other affected lymph nodes or distant metastases in order to determine disease staging and decide on the optimal surgical management.

\section{ACKNOWLEDGEMENTS}

We are indebted to Dr. Takatsugu Mizumachi, who has kindly provided us information regarding the patients of his article written in Japanese.

\section{REFERENCES}

1. Mizumachi T, Oridate N, Homma A, Nagahashi T, Furuta Y, Fukuda S, 2004 Clinical study on papillary thyroid carcinoma presenting with lymph node metastasi. Nihon Jibiinkoka Gakkai Kaiho 107: 750-755.

2. Wada N, Duh QY, Sugino K et al, 2003 Lymph node metastasis from 259 papillary thyroid microcarcinomas: frequency, pattern of occurrence and recurrence, and optimal strategy for neck dissection. Ann Surg 237: 399-407.

3. Sampson RJ, Key CR, Buncher CR, Iijima S, 1971 Smallest forms of papillary carcinoma of the thyroid. A study of 141 microcarcinomas less than $0.1 \mathrm{~cm}$ in greatest dimension. Arch Pathol 91: 334-339.

4. Zaydfudim V, Feurer ID, Griffin MR, Phay JE, 2008 The impact of lymph node involvement on survival in patients with papillary and follicular thyroid carcinoma. Surgery 144: 1070-1077.

5. Verge J, Guixá J, Alejo M et al, 1999 Cervical cystic lymph node metastasis as first manifestation of occult papillary thyroid carcinoma: report of seven cases. Head Neck 21: 370-374.

6. Levy IL, Barki Y, Tovi F, 1991 Giant cervical cyst: presenting symptom of an occult thyroid carcinoma. J Laryngol Otol 105: 863-864.

7. Loughran CF, 1991 Case report: Cystic lymph node metastasis from occult thyroid carcinoma: A sonographic mimic of a branchial cleft cyst. Clinical Radiology 42: 213-214.

8. Monchik JM, De Petris G, De Crea C, 2001 Occult papillary carcinoma of the thyroid presenting as a cervical cyst. Surgery 129: 429-432.

9. Nakagawa T, Takashima T, Tomiyama K, 2001 Differential diagnosis of a lateral cervical cyst and solitary cystic lymph node metastasis of occult thyroid papillary carcinoma. J Laryngol Otol 115: 240-242.
10. Chi HS, Wang LF, Chiang FY, Kuo WR, Lee KW, 2007 Branchial cleft cyst as the initial impression of a metastatic thyroid papillary carcinoma: two case reports. Kaohsiung J Med Sci 23: 634-638.

11. González-García R, Román-Romero L, Sastre-Pérez J, Rodríguez-Campo FJ, Naval-Gías L, 2008 Solitary cystic lymph neck node metastasis of occult thyroid papillary carcinoma. Med Oral Patol Oral Cir Bucal 13: E796-799.

12. Hwang CF, Wu CM, Su CY, Cheng L, 1992 A longstanding cystic lymph node metastasis from occult thyroid carcinoma--report of a case. J Laryngol Otol 106: 932-934.

13. Baudin E, Travagli JP, Ropers J et al, 1998 Microcarcinoma of the thyroid gland: the Gustave Roussy Institute experience. Cancer 83: 553-559.

14. Maceri DR, Babyak J, Ossakow SJ, 1986 Lateral neck mass. Sole presenting sign of metastatic thyroid cancer. Arch Otolaryngol Head Neck Surg 112: 47-49.

15. Roti E, Rossi R, Trasforini G et al, 2006 Clinical and histological characteristics of papillary thyroid microcarcinoma: results of a retrospective study in 243 patients. J Clin Endocrinol Metab 91: 2171-2178.

16. Bruno R, Giannasio P, Chiarella R et al, 2009 Identification of a neck lump as a lymph node metastasis from an occult contralateral papillary microcarcinoma of the thyroid: key role of thyroglobulin assay in the fine-needle aspirate. Thyroid 19: 531-533.

17. Gharib H, Papini E, Paschke R et al, 2010 American Association of Clinical Endocrinologists, Associazione Medici Endocrinologi, and European Thyroid Association Medical Guidelines for Clinical Practice for the Diagnosis and Management of Thyroid Nodules. Endocr Pract 16: 1-43.

18. Polyzos SA, Anastasilakis AD, 2009 Clinical complications following thyroid fine-needle biopsy: a systematic review. Clin Endocrinol (Oxf) 71: 157-165.

19. Wilczynski NL, Haynes RB, 2002 Robustness of empirical search strategies for clinical content in MEDLINE. Proc AMIA Symp 5: 904-908.

20. Appetecchia M, Meçule A, Sciarretta F, 2002 A longstanding cystic lymph-node metastasis from occult thyroid carcinoma. J Exp Clin Cancer Res 21: 137-138.

21. Francomano F, Cotellese R, Francione T, Dell'osa A, Napolitano L, 2000 Isolated cystic cervical lymphatic metastasis from occult papillary carcinoma of the thyroid: unusual and rather difficult diagnosis. G Chir 21: 327-330.

22. Mechanick JI, Camacho PM, Cobin RH et al, 2010 American Association of Clinical Endocrinologists Protocol for Standardized Production of Clinical Practice Guidelines--2010 update. Endocr Pract 16: 270-283.

23. Hay ID, Grant CS, van Heerden JA, Goellner JR, Ebersold JR, Bergstralh EJ, 1992 Papillary thyroid microcarcinoma: a study of 535 cases observed in a 50-year period. Surgery 112: 1139-1147. 
24. Davies L, Welch HG, 2006 Increasing incidence of thyroid cancer in the United States. JAMA 295: 21642167.

25. Ahuja A, Ying M, 2003 Sonography of neck lymph nodes. Part II: abnormal lymph nodes. Clin Radiol 58: 359-366.

26. Santini L, Pezzulo L, D’Arco E, De Rosa N, Guerriero O, Salza C, 1989 Lymph node metastases from an occult sclerosing carcinoma of the thyroid. A case report. Ital J Surg Sci 19: 277-279.

27. Coleman SC, Smith JC, Burkey BB, Day TA, Page RN, Netterville JL, 2000 Long-standing lateral neck mass as the initial manifestation of well-differentiated thyroid carcinoma. Laryngoscope 110: 204-209.

28. Clinberg JZ, Silver CE, Molnar JJ, Vogl SE, 1982 Cervical cysts: Cancer until proven otherwise. Laryngoscope 92: 27-30.

29. McDermott ID, Watters GW, 1996 Metastatic papillary thyroid carcinoma presenting as a typical branchial cyst. J Laryngol Otol 110: 490-492.

30. Seven H, Gurkan A, Cinar U, Vural C, Turgut S, 2004 Incidence of occult thyroid carcinoma metastases in lateral cervical cysts. Am J Otolaryngol 25: 11-17.

31. Lim DJ, Baek KH, Lee YS et al, 2007 Clinical, histopathological, and molecular characteristics of papillary thyroid microcarcinoma. Thyroid 17: 883-888.

32. The American Cancer Society. Estimated New Cancer Cases and Deaths by Sex for All Sites, US, 2010. http:// www.cancer.org/acs/groups/content/@epidemiologysurveilance/documents/document/acspc-026210.pdf(Accessed on March 7, 2012).

33. Polyzos SA, Kita M, Avramidis A, 2007 Thyroid nodules - stepwise diagnosis and management. Hormones (Athens) 6: 101-119.

34. Zhang L, Wei WJ, Ji QH et al, 2012 Risk factors for neck nodal metastasis in papillary thyroid microcarcinoma: a study of 1066 patients. J Clin Endocrinol Metab 97: 1250-1257.

35. Attie JN, Setzin M, Klein I, 1993 Thyroid carcinoma presenting as enlarged cervical lymph node. Am J Surg 166: 428-430.

36. Matsuda M, Nagumo S, Koyama H, Wada A, 1991 Occult thyroid cancer discovered by fine-needle aspiration cytology of cervical lymph node: a report of three cases. Diagn Cytopathol 7: 299-303.

37. Pazaitou-Panayiotou K, Alevizaki M, Boudina M, Drimonitis A, Kiziridou A, Vainas I, 2008 Cervical masses as manifestation of papillary thyroid carcinomas $\leq 10 \mathrm{~mm}$ in diameter, in patients with unknown thyroid disease. Thyroid Res 1: 8 .

38. Sidhu S, Lioe TF, Clements B, 2000 Thyroid papillary carcinoma in lateral neck cyst: missed primary tumour or ectopic thyroid carcinoma within a branchial cyst? J Laryngol Otol 114: 716-718.

39. Cunha N, Rodrigues F, Curado F et al, 2007 Thyroglobulin detection in fine-needle aspirates of cervical lymph nodes: a technique for the diagnosis of metastatic differentiated thyroid cancer. Eur J Endocrinol 157: 101-107.

40. Robbins RJ, Larson SM, 2008 The value of positron emission tomography (PET) in the management of patients with thyroid cancer. Best Pract Res Clin Endocrinol Metab 22: 1047-1059.

41. Al-Ashaa Y, Hefny AF, Joshi S, Abu-Zidan FM, 2011 Papillary thyroid carcinoma presenting as a lateral neck cyst. Afr Health Sci.

42. Russo D, Arturi F, Pontecorvi A, Filetti S, 1999 Genetic analysis in fine-needle aspiration of the thyroid: a new tool for the clinic. Trends Endocrinol Metab 10: 280285.

43. Fadda G, Rossi ED, Mule A, Miraglia A, Vecchio FM, Capelli A, 2006 Diagnostic efficacy of immunocytochemistry on fine needle aspiration biopsies processed by thin-layer cytology. Acta Cytol 50: 129-135.

44. Ordonez NG, 2000 Thyroid transcription factor-1 is a marker of lung and thyroid carcinomas. Adv Anat Pathol 7: 123-127.

45. Nasser SM, Pitman MB, Pilch BZ, Faquin WC, 2000 Fine-needle aspiration biopsy of papillary thyroid carcinoma: diagnostic utility of cytokeratin 19 immunostaining. Cancer 90: 307-311.

46. Chieng DC, Ross JS, McKenna BJ, 1997 CD44 immunostaining of thyroid fine-needle aspirates differentiates thyroid papillary carcinoma from lesions with nuclear grooves and inclusions. Cancer 81: 157-162.

47. Sack MJ, Astengo-Osuna C, Lin BT, Battifora H, LiVolsi VA, 1997 HBME-1 immunostaining in thyroid fineneedle aspirations: a useful marker in the diagnosis of carcinoma. Mod Pathol 10: 668-674.

48. Tovi F, Zirkin H, 1983 Solitary lateral cervical cyst: Presenting symptom of papillary thyroid adenocarcinoma. Ann Otol Rhinol Laryngol 92: 521-524.

49. Naruse T, Koike A, Kanemitsu T, Kato K, 1984 Minimal thyroid carcinoma: A report of nine cases discovered by cervical lymph node metastases. Jpn J Surg 14: 118-121.

50. Wallace MP, Betsill WL, 1984 Papillary carcinoma of the thyroid gland seen as lateral neck cyst. Arch Otolaryngol 110: 408-411.

51. Allo MD, Christianson W, Koivunen D, 1988 Not all "occult" papillary carcinomas are "minimal". Surgery 104: 971-976.

52. Park CS, Min JS, 1989 Lateral neck mass as the initial manifestation of thyroid carcinoma. Head Neck 11: 410-413.

53. Yoshida J, Nakagawa M, Yokohara K, Kishikawa H, Shiroozu A, 1990 Latent carcinoma of the thyroid manifested by cystic supraclavicular metastasis. Jpn J Clin Oncol 20: 204-208.

54. Ahuja A, Ng CF, King W, Metreweli C, 1998 Solitary cystic nodal metastasis from occult papillary carcinoma of the thyroid mimicking a branchial cyst: a potential pitfall. Clin Radiol 53: 61-63. 